\title{
Nonlinear magnetoimpedance and parametric excitation of standing spin waves in a glass-covered microwire
}

\author{
L. Kraus, ${ }^{1, a)}$ M. Vázquez, ${ }^{2}$ G. Infante, ${ }^{2}$ G. Badini-Confalonieri, ${ }^{2}$ and J. Torrejón ${ }^{2}$ \\ ${ }^{1}$ Institute of Physics, ASCR, 18221 Prague, Czech Republic \\ ${ }^{2}$ Institute of Materials Science, CSIC, 28049 Madrid, Spain
}

(Received 8 December 2008; accepted 20 January 2009; published online 9 February 2009)

\begin{abstract}
The giant magnetoimpedance of an $8.5 \mu \mathrm{m}$ glass-covered amorphous microwire was investigated in the frequency range of $10 \mathrm{MHz}-3.5 \mathrm{GHz}$. It was found that when the exciting microwave current exceeds some threshold value, a periodic fine structure appears in the frequency dependence of the complex impedance. The appearance of this nonlinear phenomenon is interpreted to be a consequence of the parametric excitation of standing spin waves. (C) 2009 American Institute of Physics. [DOI: 10.1063/1.3079659]
\end{abstract}

Nonlinear magnetization dynamics, which is one of the fundamental issues in applied magnetism, has recently received increasing attention in connection with the advances in magnetic storage technologies and spintronics. ${ }^{1}$ Materials and devices used in these areas typically have nanoscale dimensions and operate in the gigahertz range. The understanding of magnetization dynamics is of great importance for these technologies. The nonlinear behavior of ferrites and garnets was systematically investigated during the 1950s and 1960s, particularly in connection with the development of radar and microwave techniques. With increasing interest in metallic ferromagnets, nowadays, new experimental methods are desirable for investigation of the nonlinear magnetic phenomena in these materials. One such technique is the giant magnetoimpedance (GMI).

GMI comes from the dependence of electromagnetic skin depth in soft magnetic metals on the external dc magnetic field. ${ }^{2}$ It has been shown by Yelon et $_{\mathrm{al}}{ }^{3}$ that the theoretical description of GMI, which is based on the simultaneous solution of Maxwell equations and the LandauLifshitz (LL) equation of motion is similar to the theory of ferromagnetic resonance (FMR) in metals. The LL is generally a nonlinear equation. In the small signal limit (low ac excitation current), where the LL equation can be linearized, this theoretical approach provides a very good explanation for the observed GMI behavior. ${ }^{4}$ Nevertheless, at high excitation current, where the nonlinearity of LL equation must be taken into account, nonlinear effects should appear. Considering the correspondence between GMI and FMR one can expect that various nonlinear effects, similar to those observed in FMR, ${ }^{5}$ also take place in the GMI. Because of the large damping of magnetization motion, the standard nonlinear FMR experiments in ferromagnetic metals require high power microwave sources. ${ }^{6}$ In GMI measurements, however, the conditions for nonlinear behavior can already be achieved with exciting currents of a few milliampere. ${ }^{7}$ Among various nonlinear phenomena, "frequency multiplication" (higher order harmonics of GMI signal) has already been investigated. ${ }^{8-15}$ Although the similarity between the experimental configurations for transverse GMI in metallic ribbons and parallel pumping experiments in ferromagnetic insulators has already been pointed out by Yelon et al., ${ }^{7}$ the

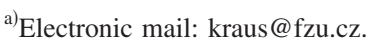

parametric excitation of spin waves, ${ }^{16}$ and other known nonlinear phenomena have not been reported so far.

The aim of this paper is to show that spatially inhomogeneous magnetic excitations can be pumped in the usual "low power" GMI microwave experiments. To demonstrate this, GMI has been measured in magnetically soft glass coated microwires which are known to exhibit quite outstanding magnetic properties, including GMI. ${ }^{17}$

The magnetoimpedance was investigated in the frequency range of $10 \mathrm{MHz}-3.5 \mathrm{GHz}$ in a glass-covered microwire prepared by the Taylor-Ulitovsky method. ${ }^{18}$ The nominal composition of the microwire alloy was $\mathrm{Co}_{67} \mathrm{Fe}_{4} \mathrm{Cr}_{7} \mathrm{Si}_{8} \mathrm{~B}_{14}$ known to exhibit nonmagnetostrictive character, determining its GMI. A piece of the sample 3.5 $\mathrm{mm}$ in length (metallic core with diameter of $8.5 \mu \mathrm{m}$ and glass coat of about $5 \mu \mathrm{m}$ thick) was mounted onto a microstrip line device under test (DUT), as schematically shown in the inset of Fig. 1. The glass coat was removed from the ends, and the sample was soldered to the line to bypass a 2.7 $\mathrm{mm}$ wide gap in the microstrip line. The distance between the electric contacts was about $2.85 \mathrm{~mm}$. The dc magnetic field of up to 100 Oe was applied parallel to the wire axis. The Agilent PNA E8362B network analyzer was used to measure the scattering parameters $S$ of the DUT. The output power was changed between -17 and $0 \mathrm{dBm}$ (i.e., $20 \mu \mathrm{W}$ $-1 \mathrm{~mW})$. The impedance $Z$ of the sample was calculated from the formula

$$
Z=R+j X=50 \frac{1-S_{11}}{1+S_{11}},
$$

where $R$ and $X$ are the resistance and reactance, respectively, and $S_{11}$ is the complex reflection parameter, corrected for the effective electrical length of the connector and the microstrip line in front of the sample. Because the impedance of sample does not match the characteristic impedance of the transmission line $(50 \Omega)$ much of the incident power is reflected before it enters the measured sample. To estimate how much microwave power passes through the sample the transmission parameter $S_{12}$ is also measured.

The frequency dependence of the real and imaginary parts of the impedance, measured at the low level of incident power $(-17 \mathrm{dBm})$ for different values of applied dc magnetic field $H$, is shown in Fig. 1. At this incident power the condition of the low signal level is well satisfied, the curves 


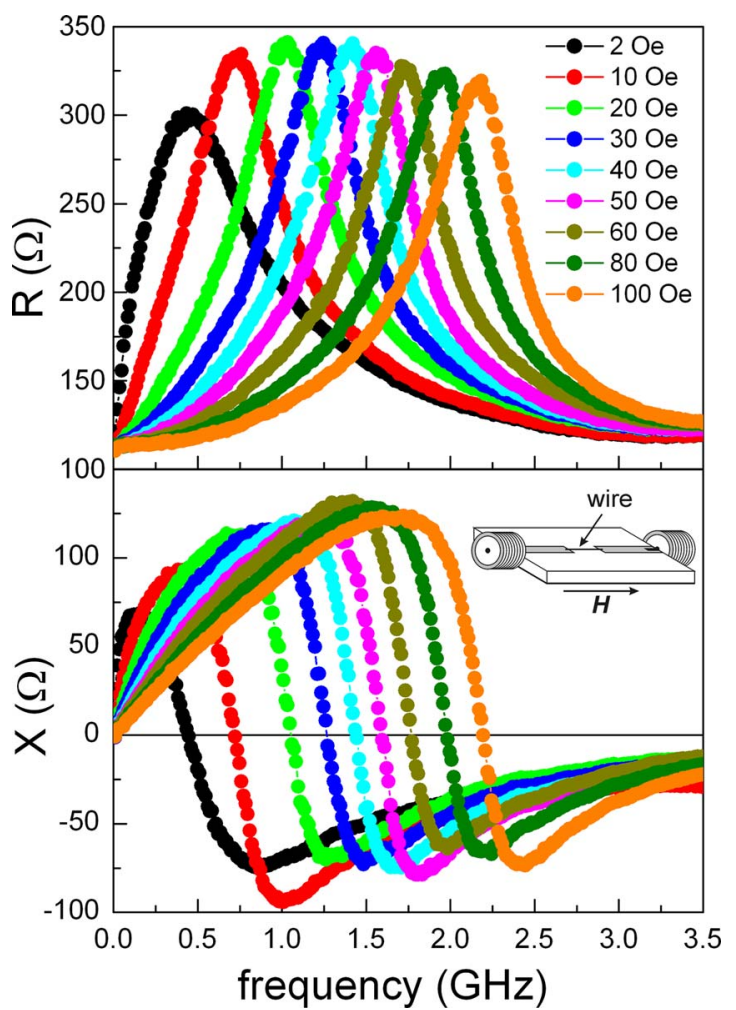

FIG. 1. (Color online) The real and imaginary parts of complex impedance for different values of applied dc field, measured at the incident microwave power of $20 \mu \mathrm{W}(-17 \mathrm{dBm})$. (Field values: 2, 10, 20, 30, 40, 50, 60, 80, and 100 Oe from the left to right.) Inset: sketch of DUT.

are smooth and show the behavior typical for FMR, i.e., maximum of $R$ and zero of $X$ at the resonance frequency. As shown in Fig. 2, the resonance frequencies $\omega$ satisfy the Kittel resonance condition for a uniaxial thin film magnetized along the in-plane easy axis ${ }^{19}$

$$
\omega / \gamma=\sqrt{\left(H+H_{K}\right)\left(H+H_{K}+4 \pi M_{s}\right)}
$$

very well. With the saturation magnetization $4 \pi M_{s}$ $=5.4 \mathrm{kG}$, determined from the hysteresis loop measurement, the experimental data can be fitted with an anisotropy field $H_{K}=2.6$ Oe and the $g$-factor of 2.09 .

When the incident microwave power increases above some critical threshold value a fine structure starts to appear on the frequency dependence of $Z$. One example of such fine structure is shown in Fig. 3, measured at constant magnetic

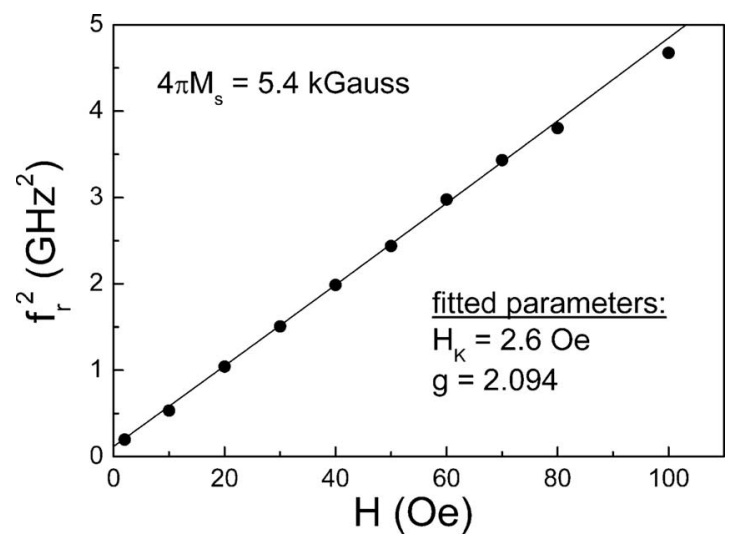

FIG. 2. Square of resonance frequency $\left(f_{r}=\omega / 2 \pi\right)$ as a function of applied field $H$ (circles denotes experimental data and line denotes theoretical fit).

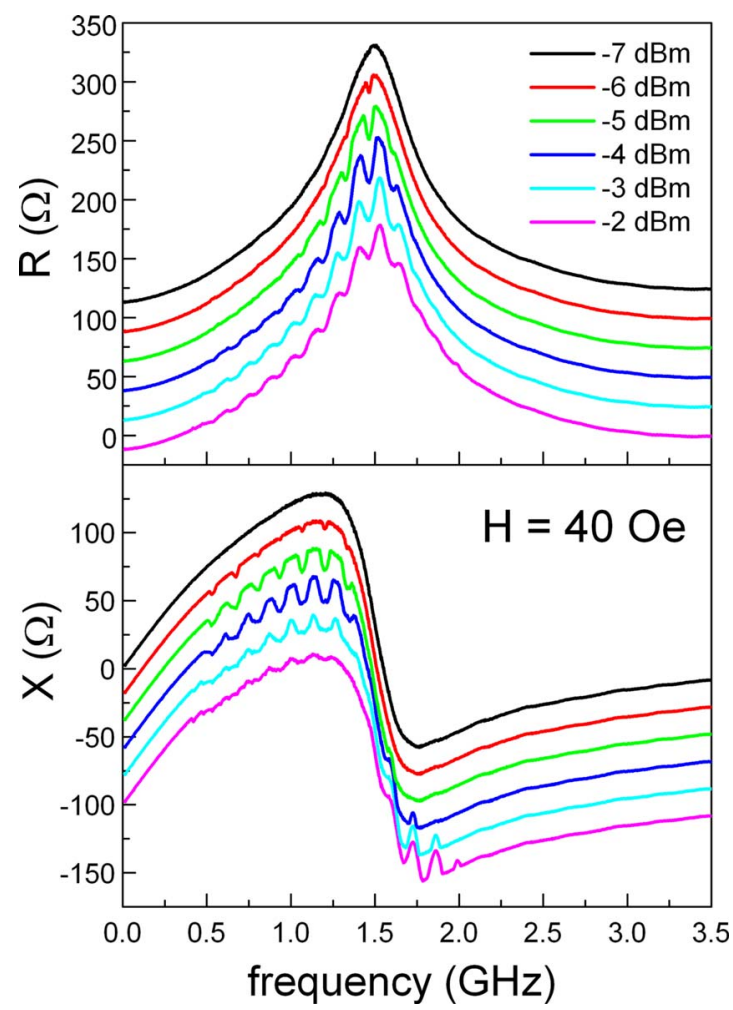

FIG. 3. (Color online) The real and imaginary parts of impedance measured under applied dc field of 40 Oe and different levels of incident microwave power. The vertical scales are valid only for the upper curves $(-7 \mathrm{dBm})$, the others are shifted downwards to distinguish the evolution of the fine structure.

field $H=40$ Oe. At an incident power of $0.2 \mathrm{~mW}(-7 \mathrm{dBm})$, the impedance is still a smooth function of frequency. When, however, this level is exceeded sharp dips appear on both the real and imaginary parts of impedance near the resonance frequency. With increasing power the dips become deeper and broader and the frequency range, where they appear, extends in both directions from the resonance. This behavior is similar to the fine structure of FMR observed in amorphous microwires ${ }^{20,21}$ and can be explained in a similar way. The threshold onset of nonlinear behavior is attributed to a spontaneous energy transfer from the basic precession mode to certain spatially nonuniform disturbances.

The theory of nonlinear FMR in ferromagnetic insulators was worked out more than 50 years ago. ${ }^{16}$ According to this theory the basic resonance mode (uniform precession in the case of ferromagnetic insulators) becomes unstable at microwave power much lower than the saturation of resonance predicted by the conventional theory. This effect, called "premature saturation of the main resonance," is caused by the decay of the uniform mode into two spin waves with opposite wave vectors $\boldsymbol{k}$ and $\boldsymbol{- k}$. Above the critical threshold, the damping of the basic resonance mode significantly increases due to the exponential growth of parametrically excited spin waves. Depending on the frequency of parametric excitations $\omega_{k}$, we speak about the first order $\left(\omega_{k}=\omega / 2\right)$ or second order $\left(\omega_{k}=\omega\right)$ Suhl instability. At low magnetic fields ( $H$ $\left.<4 \pi M_{s} / 3\right)$, where the spin wave band is degenerate with half of the pumping frequency $\omega$ (so-called "coincidence of main and subsidiary resonance"), the first order processes lead to very low critical thresholds. The critical amplitude of 
the basic resonance mode $m_{0}$ for the first order spin wave instability is given by ${ }^{20}$

$$
m_{0} / M_{s}=\eta_{\boldsymbol{k}}\left|f_{\boldsymbol{k}}\right|,
$$

where $\eta_{k}$ is the relaxation frequency and $f_{k}$ the coupling coefficient of the spin wave to the basic resonance mode. The spin waves with the lowest ratio $\eta_{k} /\left|f_{k}\right|$ are excited first, when the threshold power level is achieved.

The original Suhl theory was developed for an infinite ferromagnetic insulator, for which planar spin waves can be assumed. In finite bodies the planar waves must be replaced by the dipole-exchange modes (also called "standing spin waves"), which satisfy the surface boundary conditions. Then instead of $\boldsymbol{k}$ the pumped waves are labeled by three wave numbers $(m, n, \beta)$, which describe the quantization of the magnetization precession in three dimensions. ${ }^{22}$ In an infinite cylinder, $m$ and $n$ are related to the number of nodes in the radial and circumferential directions, respectively, and $\beta$ (any real number) is the wave vector along the axial direction. Even if the coupling coefficients $f_{m n \beta}$ were properly calculated the correct identification of the generated waves is extremely difficult, because the relaxation frequencies $\eta_{m n \beta}$ are generally unknown. In our previous paper, ${ }^{21}$ we tried to identify the fine structure peaks in the nonlinear FMR spectrum of glass-covered microwires using the exchange stiffness constant estimated from the linear spin wave resonance spectrum and the eigenfrequencies of dipole-exchange modes of an infinite insulating cylinder, theoretically investigated by Lai et al. ${ }^{23}$ This technique, however, cannot be applied here, because the exchange stiffness constant of the material under investigation is not known. Nor can the exchange constant be estimated, because the fitted value is sensitive to the proper choice of the mode numbers. To determine the actual wave vectors of the pumped spin waves, an additional experimental technique, Brillouin light scattering, has been used. ${ }^{24}$ This experiment, however, may be very difficult for the cylindrical surface micrometric radius.

It should be also mentioned that the explanation of fine structure of the "above threshold" GMI curves by the parametric excitation of spin waves need not be unique. Excitation of other waves can also be involved. The generation of acoustic waves, observed e.g., in weak ferromagnets, ${ }^{25}$ is less probable because of the low magnetostriction constant of the amorphous alloy investigated. Other excitations, inherent to ferromagnetic metals, can also account for the fine structure. To properly include the metallic nature of GMI materials further theoretical and experimental work is needed.

We have shown that with low power GMI experiments the parametric spin waves can be easily excited, which otherwise require quite intricate high power FMR equipments. This can be used as a powerful tool for the investigation of spin wave relaxation and nonlinear magnetization dynamics in metallic ferromagnets, which is now acknowledged to be of great importance for current investigations of fast switching magnetization dynamics of magnetic thin film devices.

This work was done in the frame of common project AS CR-CSIC "Amorphous and nanocrystalline soft magnetic heterostructures and their applications" and was partly supported by the Grant Agency of the Czech Republic (Grant No. 102/08/0743) and Academy of Sciences of the Czech Republic (Grant No. AVOZ 10100520), as well as by the Project No. MAT2007-65420-C02-01 supported by the Spanish Ministry of Science and Innovation.

${ }^{1}$ R. L. Stamps, J. Phys. D 38, R25 (2005).

${ }^{2}$ M. Knobel, M. Vazquez, and L. Kraus, in Giant Magnetoimpedance, edited by K. H. J. Buschow (North-Holland, Amsterdam, 2003), Vol. 15.

${ }^{3}$ A. Yelon, D. Menard, M. Britel, and P. Ciureanu, Appl. Phys. Lett. 69, 3084 (1996).

${ }^{4}$ P. Ciureanu, L. G. C. Melo, D. Seddaoui, D. Menard, and A. Yelon, J. Appl. Phys. 102, 073908 (2007).

${ }^{5}$ A. G. Gurevich and G. A. Melkov, Magnetization Oscillations and Waves (CRC, New York, 1996).

${ }^{6}$ S. Y. An, P. Krivosik, M. A. Kraemer, H. M. Olson, A. V. Nazarov, and C. E. Patton, J. Appl. Phys. 96, 1572 (2004).

${ }^{7}$ A. Yelon, M. Britel, D. Menard, and P. Ciureanu, Physica A 241, 439 (1997).

${ }^{8}$ C. Gomez-Polo, M. Knobel, K. R. Pirota, and M. Vazquez, Physica B 299, 322 (2001).

${ }^{9}$ C. Gomez-Polo, M. Vazquez, and M. Knobel, Appl. Phys. Lett. 78, 246 (2001)

${ }^{10}$ G. V. Kurlyandskaya, H. Yakabchuk, E. Kisker, N. G. Bebenin, H. GarcıaMiquel, M. Vazquez, and V. O. Vas'kovskiy, J. Appl. Phys. 90, 6280 (2001).

${ }^{11}$ G. V. Kurlyandskaya, E. Kisker, H. Yakabchuk, and N. G. Bebenin, J. Magn. Magn. Mater. 240, 206 (2002).

${ }^{12}$ G. V. Kurlyandskaya, A. Garcia-Arribas, and J. M. Barandiaran, Sens. Actuators, A 106, 234 (2003).

${ }^{13}$ J. G. S. Duque, C. Gomez-Polo, A. Yelon, P. Ciureanu, A. E. P. de Araujo, and M. Knobel, J. Magn. Magn. Mater. 271, 390 (2004).

${ }^{14}$ A. Garcia-Arribas, A. Saad, I. Orue, G. V. Kurlyandskaya, J. M. Barandiaran, and J. A. Garcia, Sens. Actuators, A 129, 275 (2006).

${ }^{15}$ D. Seddaoui, D. Menard, and A. Yelon, IEEE Trans. Magn. 43, 2986 (2007).

${ }^{16}$ H. Suhl, J. Phys. Chem. Solids 1, 209 (1957).

${ }^{17}$ M. Vazquez, in Advanced Magnetic Microwires, edited by S. Parkin and H. Kronmüller (Wiley, New Jersey, 2007), Vol. 4, p. 2193.

${ }^{18}$ A. Zhukov, J. Gonzalez, M. Vazquez, V. Larin, and A. Torcunov, in Nanocrystalline and Amorphous Magnetic Microwires, edited by H. S. Nalwa (American Scientific, California, 2003), Vol. 10, p. 522.

${ }^{19}$ P. Ciureanu, M. Britel, D. Ménard, A. Yelon, C. Akyel, M. Rouabhi, R. W. Cochrane, P. Rudkowski, and J. O. Ström-Olsen, J. Appl. Phys. 83, 6563 (1998).

${ }^{20}$ L. Kraus, Phys. Lett. A 99, 189 (1983).

${ }^{21}$ L. Kraus and M. Jirsa, J. Magn. Magn. Mater. 44, 292 (1984).

${ }^{22}$ E. Schlömann and R. I. Joseph, J. Appl. Phys. 32, 1006 (1961).

${ }^{23}$ W.-Y. Lai, D.-S. Wang, and F.-C. Pu, Acta Phys. Sin. 26, 285 (1977) [in Chinese].

${ }^{24}$ P. Kabos, G. Wiese, and C. E. Patton, Phys. Rev. Lett. 72, 2093 (1994).

${ }^{25}$ W. Wettling, W. Jantz, and C. E. Patton, J. Appl. Phys. 50, 2030 (1979). 